\title{
Formation and biological affinity evaluation of crystallographically aligned hydroxyapatite
}

\author{
Takeshi HAgIO, Tomohiko TANASE, ${ }^{*}$ Jun AKIYAMA, ${ }^{* *}$ Kazuhiko IWAI and Shigeo ASAI ${ }^{* * *}$ \\ Department of Materials, Physies and Energy Engineering, Nagoya University, Furo-cho, Chikusa-ku, Nagoya 464-8603 \\ *Toho Gas Corporation, 19-18, Sakurada-cho, Atsuta-ku, Nagoya 456-8511 \\ **Institute for Molecular Science, National Institutes of Natural Sciences, 38, Nishigonaka, Myodaiji, Okazaki 444-8585 \\ ***Innovation Plaza Tokai Japan Science and Technology Agency, 23-1, Ahara-cho, Minami-ku, Nagoya 457-0063
}

\begin{abstract}
Hydroxyapatite (HAp) is a type of calcium phosphate widely applied in the biomedical field. HAp is a main inorganic component of hard tissues of vertebrates and is a bioactive ceramic which shows a high biological affinity. The crystal of HAp exhibits an anisotropic nature depending on its crystal planes which is stemmed from its anisotropic crystal structure belonging to the hexagonal system. For instance, anions and acidic proteins are mainly adsorbed onto the a-plane of HAp crystals while cations and basic proteins are adsorbed onto the c-plane. Anisotropic nature like so can be utilized by controlling the alignment of crystals consisting the material. Alignment control of HAp crystals in a particular direction is an effective way to improve the properties like absorbability, bioactivity and biological affinity on the surface of biomedical materials. On the other hand, the recent development of superconducting technology has enabled us to control the crystal alignment of not only magnetic substances but also nonmagnetic substances with magnetic anisotropy like HAp by imposing a high magnetic field. In this study, we formed crystallographically aligned HAp bulks by using a high magnetic field and evaluated the degree of the crystal alignment. In addition, we evaluated the biological affinity on the surface of the samples.
\end{abstract}

Key-words: Hydroxyapatite, Biological affinity, Anisotropic nature, Crystal alignment, Magnetic field

[Received October 10, 2007; Accepted November 15, 2007] @2008 The Ceramic Society of Japan

1. Introduction

Hydroxyapatite (HAp) is a type of calcium phosphate widely applied in the biomedical field as bone fillers, artificial dental roots and so on. ${ }^{1)}$ HAp is a main inorganic component of hard tissues, such as bones and teeth, of vertebrates and is a nontoxic bioactive ceramic which exhibits a high biological affinity. ${ }^{2)}$ The crystal of HAp exhibits an anisotropic nature depending on its crystal planes, stemmed from its anisotropic crystal structure which belongs to the hexagonal system. Considering the adsorption nature, for example, the c-plane of HAp is an adsorption site for amino acids in proteins and for sodium ions in buffer solutions, where a phosphoric acid is the main occupant in the crystal plane. Meanwhile, the a-plane is the favored adsorption sites for carboxyl groups in proteins, and for phosphoric acids in buffer solutions, because calcium is the main occupant in the crystal plane. ${ }^{3), 4)}$ For this reason, controlling the crystal alignment of HAp crystals consisting the material makes is possible for us to enhance and utilize such anisotropic nature.

Observing the structure of the actual long bones of humans, the HAp nano crystals consisting it forms a selforganized structure so that the $c$-axis of the HAp crystal becomes parallel to the direction of the maximum load. ${ }^{5)}$ The structure of things in nature has been selectively formed through its long history so this means understanding the basis of the actual structure of hard tissues of vertebrates is very important for the formation of a more advanced material for medical applications. For this reason, we can say that controlling the alignment of HAp crystals in a direction resembling the actual hard tissue has efficacy for improving and optimizing various properties, such as absorbability, bioactivity and biological affinity, of biomedical materials. However, the difference in properties of each crystal plane of HAp has not been investigated yet.

On the other hand, the recent development of superconducting technology has enabled us to control the crystal alignment of non-magnetic substances with magnetic anisotropy like HAp by imposing a high magnetic field. ${ }^{6)-8)}$

In this study, formation of crystallographically aligned HAp bulks in both $a$-axis and $c$-axis direction has been performed using a high magnetic field. Then the degree of crystal alignment and the biological affinity on the surface of each sample was evaluated by immersing them into the simulated body fluid (SBF) proposed by Kokubo et al..9),10)

\section{Method of crystal alignment using a magnetic field}

The principle of crystal alignment for non-magnetic materials using a magnetic field can be explained by the generation of the magnetization energy. When a magnetic field is applied to a non-magnetic material, the generated magnetization energy per unit volume $U$ is approximately indicated as

$$
U \cong-\frac{1}{2} \mu_{0} \chi H^{2}
$$

where $\mu_{0}$ is the magnetic permeability in vacuum, $\chi$ is the magnetic susceptibility parallel to the magnetic field, and $H$ is the intensity of the external magnetic field, respectively.

Now, when we consider a magnetically anisotropic crystal placed in a magnetic field, the magnetization energy will differ according to the direction of the magnetic field applied to the crystal. Thus the crystal rotates in the direction that reduces the magnetization energy. This continuously occurs 


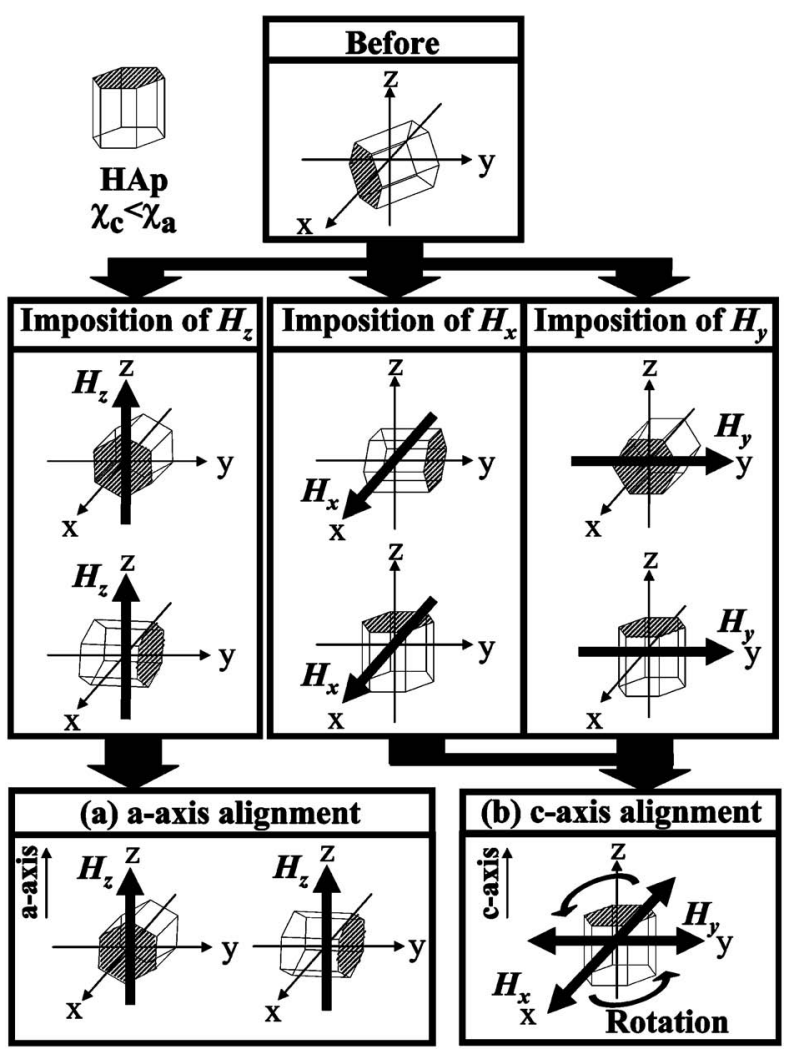

Fig. 1. Principle of crystal alignment using a magnetic field. (a) crystal aligned in the $a$-axis direction seen from the $z$-axis direction, (b) crystal aligned in the $c$-axis direction seen from the $z$-axis direction.

until the crystal settles to a stabilized state. As for HAp crystals, the magnetic susceptibility is expected to be $\chi_{\mathrm{c}}<\chi_{\mathrm{a}}$ from the previous study. ${ }^{11)}$ This information not only indicates that alignment of HAp crystals in the $a$-axis direction is obtainable by the imposition of a static magnetic field as shown in Fig. 1 (a) but also that alignment of HAp crystals in the $c$-axis direction is unobtainable by using a static magnetic field. To enable crystal alignment in the $c$-axis direction, a simultaneous imposition of the static magnetic field and mold rotation has been carried out. ${ }^{11)-13)}$ The method of crystal alignment in the $c$-axis direction using this method is based on the following principle. To begin with, assume that a magnetic field was imposed on a crystal in the $x$-axis direction. This limits the $c$-axis of the crystal to rotate only in the $y-z$ plane, since it is the stable condition when the magnetization energy is considered. On the other hand, if we assume that a magnetic field was imposed on the crystal in the $y$-axis direction, the $c$-axis of the crystal will rotate only in the $x-z$ plane in the same reason explained before. Therefore, by imposing a magnetic field in the $x$-axis and $y$-axis direction simultaneously or alternately, we can confine the $c$-axis of the HAp crystal in a single direction, the $z$-axis direction. This alternate imposition of the magnetic field can be attained by imposing a rotating magnetic field as shown in Fig. 1 (b). This can be achieved relatively by the simultaneous imposition of a static magnetic field and sample rotation in the $x-y$ plane.
Table 1. Experimental Conditions for the Slip Cast Process

\begin{tabular}{cccc}
\hline Sample No. & $\begin{array}{c}\text { Magnetic Flux } \\
\text { Density [T] }\end{array}$ & $\begin{array}{c}\text { Direction of } \\
\text { Magnetic Field Applied }\end{array}$ & $\begin{array}{c}\text { Mold Rotation } \\
{[\mathbf{r a d} / \mathbf{s}]}\end{array}$ \\
\hline 1 & 0 & - & - \\
2 & 10 & $\begin{array}{c}\text { perpendicular to bottom } \\
\text { surface of the mold } \\
\text { parallel to bottom } \\
\text { surface of the mold }\end{array}$ & - \\
3 & 10 & 0.3 \\
\hline
\end{tabular}

\section{Experimental procedure}

3.1 Formation of crystal aligned HAp bulk

A slurry prepared by mixing $15 \mathrm{~g}$ of HAp particles (Taihei Chemical Co., HAp-200) and about $15 \mathrm{~g}$ of ion-exchanged water and a small amount of deflocculant was milled mechanically for more than 4 hours and poured into a gypsum mold. Then the mold was set inside the super conducting magnet to impose a high magnetic field during the slip casting process. In this experiment, the slip casting process was proposed under three different conditions; without a magnetic field, with a static magnetic field, and with a relative rotating magnetic field. The three conditions are shown in Table 1. After slip cast, the samples were dried in room temperature for more than $24 \mathrm{~h}$ without a magnetic field and were sintered at $1573 \mathrm{~K}$ in an electric furnace. The samples were analyzed before and after the sintering by using the X-ray diffractometer (Rigaku Corp., cat. no. 2035G403) with a $\mathrm{Cu} \mathrm{K} \alpha \mathrm{X}$-ray source. The degree of crystal alignment was evaluated by the resultant X-ray diffraction (XRD) patterns.

\subsection{Biological affinity evaluation}

After confirming that crystallographically aligned samples have been formed successfully by using the method explained before, the samples were polished and then immersed into the SBF. The biological affinity of the surface of each sample was evaluated by the thickness of the precipitate observed on the surface of the samples after 12 , 24 and $48 \mathrm{~h}$ from the SBF immersion.

\section{Results and discussion}

4.1 Degree of crystal alignment of HAp

The XRD patterns of the samples obtained under the three different experimental conditions are shown in Fig. 2. The three on the left are the samples before the sintering and the three on the right are the ones after the sintering. The diffraction pattern of sample 1 before the sintering is nearly the same with the diffraction pattern of HAp powder reported in the JCPDS card \#9-432 and it indicates that the crystals are randomly aligned. On the other hand, the diffraction patterns of samples 2 and 3 before the sintering show that the peak intensities indicating the $a$-planes relatively increased and those of $c$-planes relatively decreased in the sample 2 while the peak intensities indicating the $c$-planes relatively increased and those of $a$-planes relatively decreased in the samples. In fact, the intensity ratio of $(002) /(300)$ calculated in samples 1, 2, 3 before the sintering were $0.477,0.089$, 3.21 , respectively. This indicates that crystal alignment has been performed successfully. Furthermore, the diffraction pattern of the sample 1 after the sintering showed no big difference compared to the patterns before the sintering while the degree of crystal alignment in the sample 2 has been 


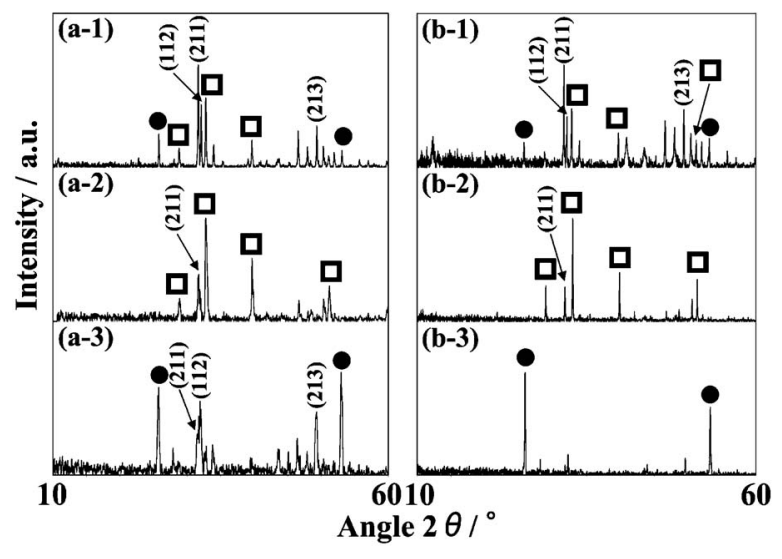

Fig. 2. XRD patterns of the samples before and after the sintering. : $c$-plane $\square$ : $a$-plane

(a-1) without magnetic field, before the sintering, (a-2) with static magnetic field, before the sintering, (a-3) with both static magnetic field and mold rotation, before the sintering, (b-1) without magnetic field, after the sintering, (b-2) with static magnetic field, after the sintering, (b-3) with both static magnetic field and mold rotation, after the sintering.

slightly improved in the $a$-axis direction. Furthermore, obvious improvement was seen in the $c$-axis direction in the sample 3. Comparing the XRD patterns of Fig. 2(a-3) and (b-3), the peaks which do not indicate neither the $a$-plane or the $c$-plane such as (211), (112) and (213) has nearly disappeared after the sintering process. This improvement in the degree of crystal alignment seen in the sintering process of sample 3 can be understood by the interfacial energy between the crystals. ${ }^{14), 15)}$ Since the interfacial energy between uniaxially aligned crystals is smaller than that between nonaligned crystals, the grain growth of uni-axially aligned crystals preferentially occurred compared to the non-aligned crystals during the sintering process. From this result, we can say the sintering process is an effective way to enhance the degree of crystal alignment of HAp crystals in samples already aligned to a certain extent.

\subsection{Biological affinity of crystallographically Aligned} HAp

The biological affinity of the surface of the samples was evaluated by immersing them into the SBF. Figure 3 shows the SEM images of the two crystallographically aligned samples, in the $a$-axis direction (sample 2) and $c$-axis direction (sample 3) immersed in the SBF for $48 \mathrm{~h}$. The deposition of the precipitate can be recognized in both crystallographically aligned samples but the thickness of the precipitate observed on the sample aligned in the $c$-axis direction (sample 3 ) was much thicker compared to that on the sample aligned in the $a$-axis direction (sample 2). This indicates that the ability to produce the precipitate differs depending on crystal planes of HAp. Table 2 shows the thickness of the precipitate observed on the three samples at $0,12,24$ and $48 \mathrm{~h}$ after the immersion in SBF. From this table, it can be said that the thickness of the precipitate was thickest on the surface of the sample aligned in the $c$-axis direction (sample 3 ) and became thinner on the sample that was not aligned (sample 1) and thinnest on the sample aligned in the $a$-axis direction (sample 2 ). In addition, we can say that the time precipitate depo-
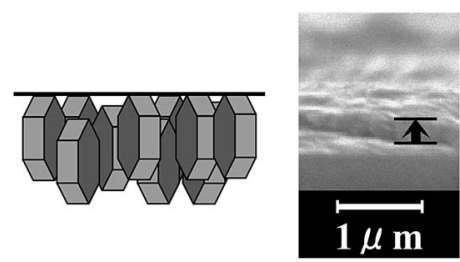

(a)
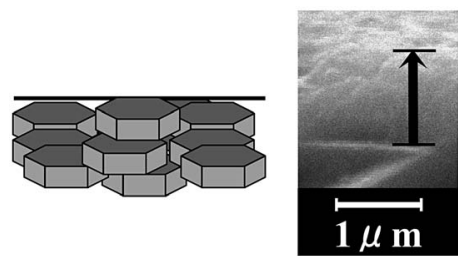

(b)

Fig. 3. SEM images of the cross-section of crystallographically aligned samples immersed in SBF for $48 \mathrm{~h}$.

(a) precipitate observed on the surface of the sample crystallographically aligned in the $a$-axis direction, (b) precipitate observed on the surface of the sample crystallographically aligned in the $c$-axis direction.

Table 2. Thickness of Precipitate Observed on Top Surface of Samples after SBF Immersion

\begin{tabular}{cccc}
\hline $\begin{array}{c}\text { Immersing } \\
\text { period in SBF } \\
\text { / hour }\end{array}$ & \multicolumn{3}{c}{ Thickness of Precipitate on the Top Surface $/ \mu \mathbf{m}$} \\
\cline { 2 - 4 } & $\begin{array}{c}\text { random } \\
\text { sample 1 }\end{array}$ & $\begin{array}{c}\text { a-axis alignment } \\
\text { sample 2 }\end{array}$ & $\begin{array}{c}\text { c-axis alignment } \\
\text { sample 3 }\end{array}$ \\
\hline 0 & 0 & 0 & 0 \\
12 & 0.067 & 0 & 0.14 \\
24 & 0.23 & 0 & 1.3 \\
48 & 0.62 & 0.28 & 1.1 \\
\hline
\end{tabular}

sited on the surface of the samples differs. The difference observed here may be explained by crystal planes dependence of growth rate and growth mechanism of HAp. Onuma et al. ${ }^{16)-18)}$ observed the crystal growth and measured the growth rate of the (100) and (001) plane, which corresponds to the $a$-plane and $c$-plane of hydroxyapatite single crystals. As a result, they found out that growth rate of the $c$-plane was larger than that of the $a$-plane and also found the growth mechanisms depending on the planes. The growth mechanism of the $a$-plane was step flow combined with two-dimensional nucleation ${ }^{16), 17)}$ while that of the $c$ plane was a multiple two-dimensional nucleation. ${ }^{17), 18)}$ The same phenomena could have taken place in this study.

\section{Conclusion}

We proposed a crystal alignment method using a magnetic field to form HAp bulk with crystal alignment. From the XRD patterns, it has been found that crystal alignment in a particular direction can be achieved in the slip casting process by imposing a static magnetic field and mold rotation selectively. It has also been found that a high degree of crystal alignment can be obtained by sintering a sample which is already crystallographically aligned to some extent. On the other hand, from the thickness of the precipitate on the surface of the samples immersed in SBF for a particular 
period, it has been found that there is difference in the deposition time and thickness depending on crystal planes of HAp. The control of crystal alignment may make it possible for us to form a more advanced biomaterial.

Acknowledgement This work was partially supported by JSPS Asian Core Program "Construction of the World Center on Electromagnetic Processing of Materials."

\section{References}

1) H. Aoki, "Medical Applications of Hydroxyapatite," Ishiyaku EuroAmerica, Inc., Tokyo, St. Louis (1994) pp. 1-12, 90100, 156-173.

2) R. Z. LeGeros and J. P. LeGeros, "An Introduction to Bioceramics,” World Scientific (1993) 139.

3) T. Kawasaki, J. of Chromatography, 151, 105 (1978).

4) T. Kawasaki, M. Nimura and Y. Kobayashi, J. of Chromatography, 515, 91 (1990).

5) T. Nakano, K. Kaibara, Y. Tabata, N. Nagata, S. Enomoto, E. Marukawa and Y. Umakoshi, Bone, 31, 479 (2002).

6) H. Morikawa, K. Sassa and S. Asai, Mater. Trans., 39, 814-818 (1998).

7) T. Suzuki, Y. Sakka and K. Kitazawa, J. Ceram. Soc. Japan, 109, 886-890 (2001).
8) S. Asai, K. Sassa and M. Tahashi, Sci. Technol. Adv. Mater., 4, 455-460 (2001).

9) T. Kokubo, H. Kushitani and S. Sakka, J. Biomed. Mater. Res., 24, 721 (1990).

10) T. Kokubo and H. Kushitani, Biomaterials, 27, 2907 (2006).

11) K. Inoue, T. Marukawa, K. Sassa, Y. Yokogawa, Y. Sakka, M. Okido and S. Asai, Key Eng. Mater., 240-242, 513-516 (2003).

12) K. Inoue, K. Sassa, Y. Yokogawa, Y. Sakka, M. Okido and S. Asai, Mater. Trans., 44, 1133-1137 (2003).

13) J. Akiyama, M. Hashimoto, H. Takadama, F. Nagata, Y. Yokogawa, K. Sassa, K. Iwai and S. Asai, Mater. Trans., 46, 2, 203-206 (2005).

14) J. Akiyama, M. Hashimoto, H. Takadama, F. Nagata, Y. Yokogawa, K. Sassa, K. Iwai and S. Asai, Mater. Trans., 46, 2514-2517 (2005).

15) T. Nakano, W. Fujitani and Y. Umakoshi, Mater. Sci. Forum. 449-452, 1289-1292 (2004).

16) K. Onuma, A. Ito, T. Tateishi and T. Kameyama, J. Cryst. Growth, 154, 118-125 (1995).

17) N. Kanzaki, K. Onuma, A. Ito, K. Teraoka, T. Tateishi and S. Tsutsumi, J. Phys. Chem. B, 102, 6471-6476 (1998).

18) K. Onuma, N. Kanzaki, A. Ito and T. Tateishi, J. Phys. Chem. B, 102, 7833-7838 (1998). 